ARTICLE

\title{
Defect-implantation for the all-electrical detection of non-collinear spin-textures
}

\author{
Imara Lima Fernandes (id ${ }^{1 \times}$, Mohammed Bouhassoune $e^{1} \&$ Samir Lounis ${ }^{1 \times}$
}

The viability of past, current and future devices for information technology hinges on their sensitivity to the presence of impurities. The latter can reshape extrinsic Hall effects or the efficiency of magnetoresistance effects, essential for spintronics, and lead to resistivity anomalies, the so-called Kondo effect. Here, we demonstrate that atomic defects enable highly efficient all-electrical detection of spin-swirling textures, in particular magnetic skyrmions, which are promising bit candidates in future spintronics devices. The concomitant impurity-driven alteration of the electronic structure and magnetic non-collinearity gives rise to a new spin-mixing magnetoresistance $\left(\mathrm{XMR}_{\text {defect }}\right)$. Taking advantage of the impuritiesinduced amplification of the bare transport signal, which depends on their chemical nature, a defect-enhanced XMR (DXMR) is proposed. Both XMR modes are systematised for $3 d$ and $4 d$ transition metal defects implanted at the vicinity of skyrmions generated in PdFe bilayer deposited on $\operatorname{Ir}(111)$. The ineluctability of impurities in devices promotes the implementation of defect-enabled XMR modes in reading architectures with immediate implications in magnetic storage technologies.

\footnotetext{
${ }^{1}$ Peter Grünberg Institut and Institute for Advanced Simulation, Forschungszentrum Jülich and JARA, D-52425 Jülich, Germany

凶email: i.lima.fernandes@fz-juelich.de; s.lounis@fz-juelich.de
} 
D efects are inherent to all devices and materials. Being unavoidable, they dramatically reshape transport properties, often negatively, and thus are key ingredients in settling the competitiveness of newly proposed technologies and therefore their survival. In the context of spintronics ${ }^{1}$ exploiting the spin rather than the charge to carry information, defects can reduce the efficiency of magnetoresistance effects ${ }^{2,3}$ in current perpendicular-to-plane geometries such as the giant magnetoresistance $(\mathrm{GMR})^{4,5}$ or tunneling magnetoresistance (TMR) ${ }^{6,7}$. Impurities intrinsically can alter the conductance by increasing it or reducing it, as shown for various constrictions ${ }^{8-11}$, giving rise to inelastic transport channels allowing the exploration of electron-bosons interactions ${ }^{12-14}$ while generating extrinsic contributions to Hall effects ${ }^{15-20}$ in current-in-plane geometries. All of this is not surprising since the very fundamental Kondo effect $^{21}$ results from diluted magnetic impurities leading to an anomalous behavior of the resistance at low temperature ${ }^{22}$.

Recently a new kind of magnetoresistance effect, the spinmixing magnetoresistance (XMR), has been discovered ${ }^{23,24}$, which in contrast to the GMR or TMR effect enables the allelectrical detection of non-collinear magnetic states, such as magnetic skyrmions ${ }^{25,26}$ and spin spirals, with a non-magnetic electrode. Thus, XMR provides an appealing detection tool of importance in establishing spin-swirling textures with chiral or topological protection properties, in particular skyrmions, as future bits for information technology 27 .

While several investigations were devoted to the impact of inhomogeneities on the motion and stability of skyrmions ${ }^{28-39}$, their implications in their electrical detection are yet to be determined. Similarly to the GMR and TMR effects, it is often expected that defects would reduce the XMR efficiency.

In this article, we demonstrate from a full ab initio approach that contrary to the current wisdom atomic defects are of technological importance in reading non-collinear spin-states since they enable a highly efficient spin-mixing magnetoresistance signal, where the reference transport signal is amplified by many of the investigated impurities (see Fig. 1a). We envision scenarios, where impurities are manipulated atom-by-atom ${ }^{40}$, implanted ${ }^{41}$ or spontaneously generated via intermixing mechanisms. We introduce defect-enabled XMR modes in order to evaluate the potential of defects in magnifying simultaneously the transport and XMR signals. We perform systematic simulations of atomic resolved transport measurements as probed within scanning tunneling microscopy/spectroscopy (STM/STS) of $3 d$ (V, Cr, Mn, $\mathrm{Fe}, \mathrm{Co}, \mathrm{Ni}$ ) and $4 d(\mathrm{Nb}, \mathrm{Mo}, \mathrm{Tc}, \mathrm{Ru}, \mathrm{Rh})$ transition metal defects implanted in the Pd surface layer covering the fcc-Fe monolayer deposited on $\operatorname{Ir}(111)$ surface. The latter substrate is known to host few nanometers-wide magnetic skyrmions ${ }^{42-47}$ stabilized by the presence of Dzyaloshinskii-Moriya interaction ${ }^{48,49}$. We identified the different mechanisms conspiring to make the universal trends of the various XMRs, as function of the impurities electronic states, distinct from each other. We put forward the defectenabled XMR effects as key-reading tools in man-engineered defective substrates with immediate implications for device applications in the context of non-collinear states.

\section{Results}

Impact of defects on the all-electrical detection. The allelectrical detection of single magnetic skyrmions via the XMR effect $^{23,24,50}$ is made possible via the non-collinearity of the magnetic moments of the Fe layer and the presence of spin-orbit interaction, which lead to a spin-mixing of the electronic states living initially in the majority- and minority-spin channels. Within tunneling transport experiments, these mixed states are detectable with a non-magnetic electrode (Fig. 1a). In the case of
$\mathrm{PdFe} / \mathrm{Ir}(111)$ substrate, the XMR effect as probed in STM/STS is particularly enhanced for resonant interface and surface states ${ }^{51}$, which result from various hybridization mechanisms being affected by relativistic effects.

We consider a skyrmion passing at the vicinity of a defect as illustrated schematically in Fig. 1. Here, it is convenient to define two types of XMR signals contingent on the reference measurement with respect to which the efficiency of the MR effect is calculated. The reference point can be either the clean (defectfree) (see Fig. 1b) or the substrate-defect complex (see Fig. 1c), both in the ferromagnetic configuration. The former leads to the defect-enhanced XMR (DXMR) while the latter is a XMR signal obtained in the presence of the defect and denoted in the following $\mathrm{XMR}_{\text {defect }}$. In both cases, we calculate the percent deviation of the conductance on top of a skyrmion from that of a reference point within a collinear magnetic region. In a device, such reference points have to be identified, for instance, after saturating the magnetic state by applying a magnetic field. Then the regions with and without defects can be prospected and identified through the change in the transport signal. We note that the XMR signal collected from an STM experiment on a pinned skyrmion ${ }^{24}$ naturally includes the contribution of the pinning defect and should thus correspond to what we name DXMR.

Based on the Tersoff-Hammann approach ${ }^{52}$, the differential conductance $\frac{\mathrm{dI}}{\mathrm{dV}}$ at a given bias voltage $V_{\text {bias }}$ measured within STM/STS, equipped with a non-magnetic tip, is proportional to the local density of states (LDOS) of the substrate at the energy $E=E_{\mathrm{F}}+e V_{\text {bias }}$ obtained in vacuum at the tip position. While our focus is the differential conductance, the definitions given below could be extended to the constant-current mode of STM (see Supplementary Note 1). Here, we consider the tip located in second vacuum layer above the Pd substrate as indicated in Fig. 1b, c, thus the efficiency of these magnetoresistance effects are calculated from:

$$
\mathrm{XMR}_{\text {defect }}(E)=\frac{\operatorname{LDOS}_{\mathrm{NC}}^{\text {defect }}(E)-\operatorname{LDOS}_{\mathrm{C}}^{\text {defect }}(E)}{\operatorname{LDOS}_{\mathrm{C}}^{\text {defect }}(E)},
$$

which is analogue to the conventional defect-free XMR,

$$
\mathrm{XMR}_{\text {clean }}(E)=\frac{\operatorname{LDOS}_{\mathrm{NC}}^{\text {clean }}(E)-\operatorname{LDOS}_{\mathrm{C}}^{\text {clean }}(E)}{\operatorname{LDOS}_{\mathrm{C}}^{\text {clean }}(E)},
$$

where all LDOS are obtained in the clean region. $\mathrm{C}$ and $\mathrm{NC}$ correspond respectively to collinear and non-collinear magnetic regions, as shown in Fig. $1 \mathrm{~b}, \mathrm{c}$. It is important to note that $\mathrm{XMR}_{\text {defect }}$ and $\mathrm{XMR}_{\text {clean }}$ effects are fundamentally different since the former is settled by the defect's electronic states and how they react to the presence of a non-collinear spin-texture.

The proposed DXMR efficiency is extracted from:

$$
\begin{array}{r}
\operatorname{DXMR}(E)=\frac{\operatorname{LDOS}_{\mathrm{NC}}^{\text {defect }}(E)-\operatorname{LDOS}_{\mathrm{C}}^{\text {clean }}(E)}{\operatorname{LDOS}_{\mathrm{C}}^{\text {clean }}(E)} \\
=\mathrm{XMR}_{\text {clean }}(E)+\frac{\operatorname{LDOS}_{\mathrm{NC}}^{\text {defect }}(E)-\operatorname{LDOS}_{\mathrm{NC}}^{\text {clean }}(E)}{\operatorname{LDOS}_{\mathrm{C}}^{\text {clean }}(E)},
\end{array}
$$

which obviously measures the enhancement of the XMR efficiency by the defect with respect to the signal obtained in the defect-free region. The latter equation shows that the DXMR signal is related to the "traditional" defect-free XMR effect and it is obviously shaped by how the tunneling matrix elements involving the defect are different from those of the defect-free substrate. In fact, by re-expressing it as:

$$
\begin{aligned}
1+\operatorname{DXMR}(E) & =\left(1+\operatorname{XMR}_{\text {defect }}(E)\right) \cdot\left(1+D_{\mathrm{C}}(E)\right) \\
& =\left(1+\operatorname{XMR}_{\text {clean }}(E)\right) \cdot\left(1+D_{\mathrm{NC}}(E)\right),
\end{aligned}
$$




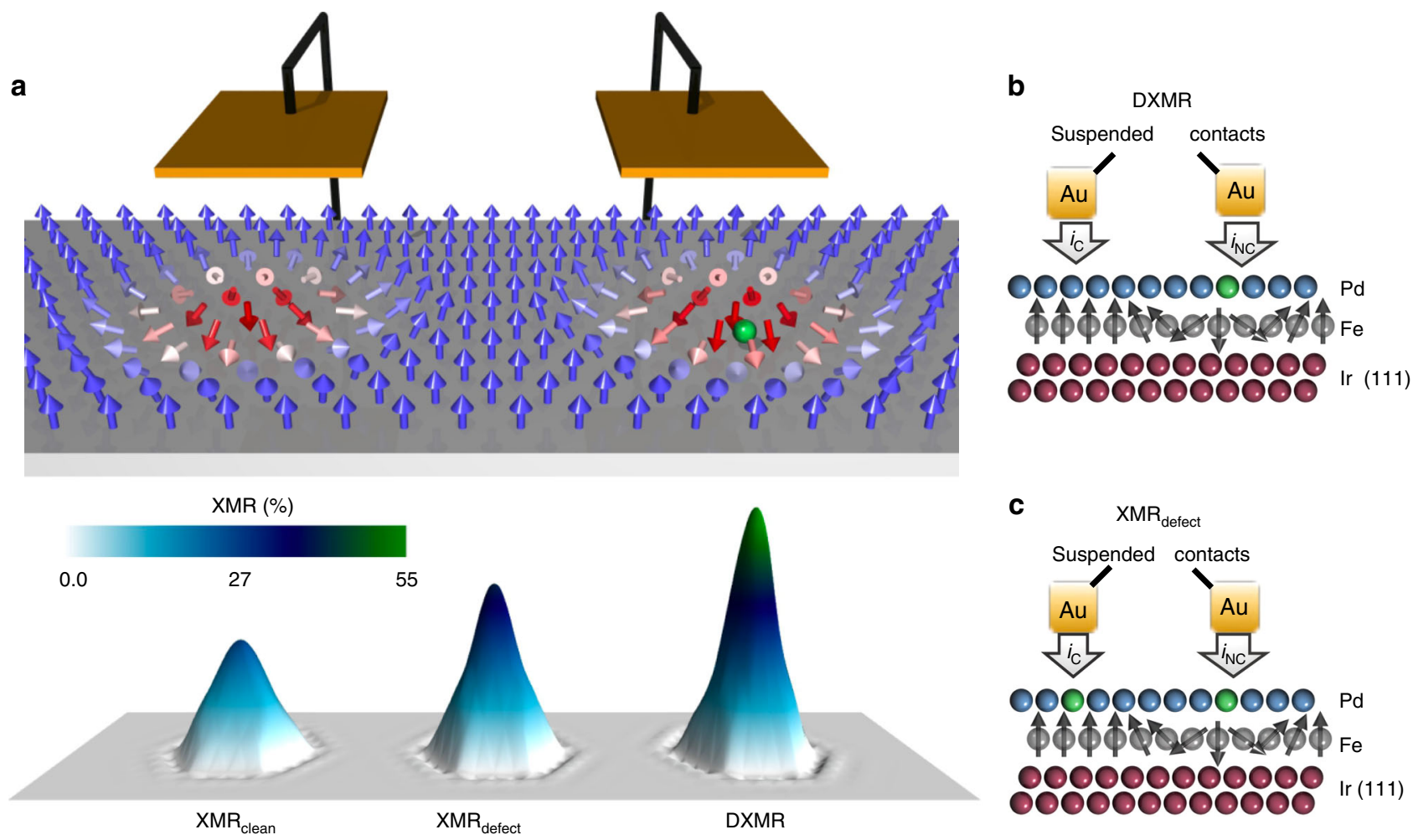

Fig. 1 All-electrical detection of magnetic skyrmions in the presence of defects. a Illustration of a tunneling transport experiment to read magnetic skymions on fcc-PdFe bilayer on the $\operatorname{Ir}(111)$ surface away from (left) or at the vicinity of (right) a single-atomic defect indicated by a green sphere. The spinmixing of the electronic structure within the non-collinear environment leads to changes on the tunneling conductance allowing the magnetic data information to be sensed in a current perpendicular-to-plane geometry. The various XMRs signals, for the clean system and in the presence of defects, are shown on the lower panel. Depending on the reference background two types of XMR signals can be measured, $\mathbf{b}$ while the clean ferromagnetic substrate leads to the defect-enhanced XMR (DXMR), $\mathbf{c}$ the substrate-defect complex background leads to the $X M R_{\text {defect. }}$.

where

$$
D_{\mathrm{NC}}(E)=\frac{\operatorname{LDOS}_{\mathrm{NC}}^{\text {defect }}(E)-\operatorname{LDOS}_{\mathrm{NC}}^{\text {clean }}(E)}{\operatorname{LDOS}_{\mathrm{NC}}^{\text {clean }}(E)}
$$

and

$$
D_{\mathrm{C}}(E)=\frac{\operatorname{LDOS}_{\mathrm{C}}^{\text {defect }}(E)-\operatorname{LDOS}_{\mathrm{C}}^{\text {clean }}(E)}{\operatorname{LDOS}_{\mathrm{C}}^{\text {clean }}(E)}
$$

one deduces that the DXMR signal can be interpreted as the $\mathrm{XMR}_{\text {defect }}$ signal altered by the bare signal provided by the defect. The larger the latter is, the better the detection of the magnetic skyrmion. We note that $D_{\mathrm{C}}$ can be obtained in the saturared magnetic state prior to the generation or injection of the noncollinear spin-textures.

For conciseness, we focus our first analyses on the impact of a $\mathrm{V}$ impurity at the closest vicinity of the skyrmion's core on the various XMRs, whose energy-resolved signals are plotted in Fig. 2a. The investigated skyrmion has a diameter $D_{\mathrm{Sk}} \approx 2.2 \mathrm{~nm}$ and we recall that in the skyrmion's core, the magnetic moment of the substrate $\mathrm{Fe}$ atom is flipped with respect to the ferromagnetic surrounding. Among the XMR signals, the DXMR efficiency is the largest with an impressive $\approx 85 \%$, i.e., an increase of $\approx 230 \%$ with respect to the defect-free signal, for an energy injection $e V_{\text {bias }}=+0.45 \mathrm{eV}$ (Fig. 2a, red curve). As deduced from Eq. (4), this large efficiency is just an indication that the defect enhances the bare tunneling transport signal. For comparison, the $\mathrm{XMR}_{\text {clean }}$ (Fig. 2a, green curve) and $\mathrm{XMR}_{\text {defect }}$ (Fig. 2a, blue curve) signals reach a similar efficiency around $26 \%$. In the whole investigated energy window, the latter two signals have a shape, which in general, seems similar to the
DXMR signal. Moreover, we note that the large efficiencies observed in the various XMR effects obtained on the basis of the differential conductance measurements are present in the constant-current mode (see Supplementary Note 1).

The $\mathrm{XMR}_{\text {clean }}$ efficiency is shaped by the non-collinearity of the substrate magnetic moments and the presence of spin-orbit interaction. Two additional mechanisms, enabled by the presence of the defect, contribute to the $\mathrm{XMR}_{\text {defect: }}$ (i) impurity-induced modification of the substrate's non-collinearity and (ii) hybridization of the electronic states of the defect with the substrate $\mathrm{Fe}$ atoms and the way these states decay into vacuum, which settles the tunneling matrix elements. In contrast, the DXMR signal is more complex since it involves mechanisms active for $\mathrm{XMR}_{\text {clean }}$ and $\mathrm{XMR}_{\text {defect }}$ weighted by the enhancement factors introduced by the defect. The latter would be useful to enhance weak transport signals that can be of importance for buried skyrmions located away from the probing electrodes.

Enhancement of the bare signal and of the DXMR efficiency. The reference background for both the $\mathrm{XMR}_{\text {clean }}$ and the DXMR signal is the defect-free region away from the skyrmion (collinear magnetic region). Thus, to understand the enhancement of the DXMR effect, we invoke Eq. (4) and analyse the vacuum LDOS just above the skyrmion core with and without the implanted Vatom (Fig. 2e, f). As illustrated in Fig. 2b, the vacuum electronic states of both spin-channels are strongly amplified by the presence of the V-defect affecting the differential conductance by virtue of the Tersoff-Hamann approximation (see Supplementary Note 2 for details related to the electronic structure of the collinear magnetic region). Here one identifies the main advantage of 
a
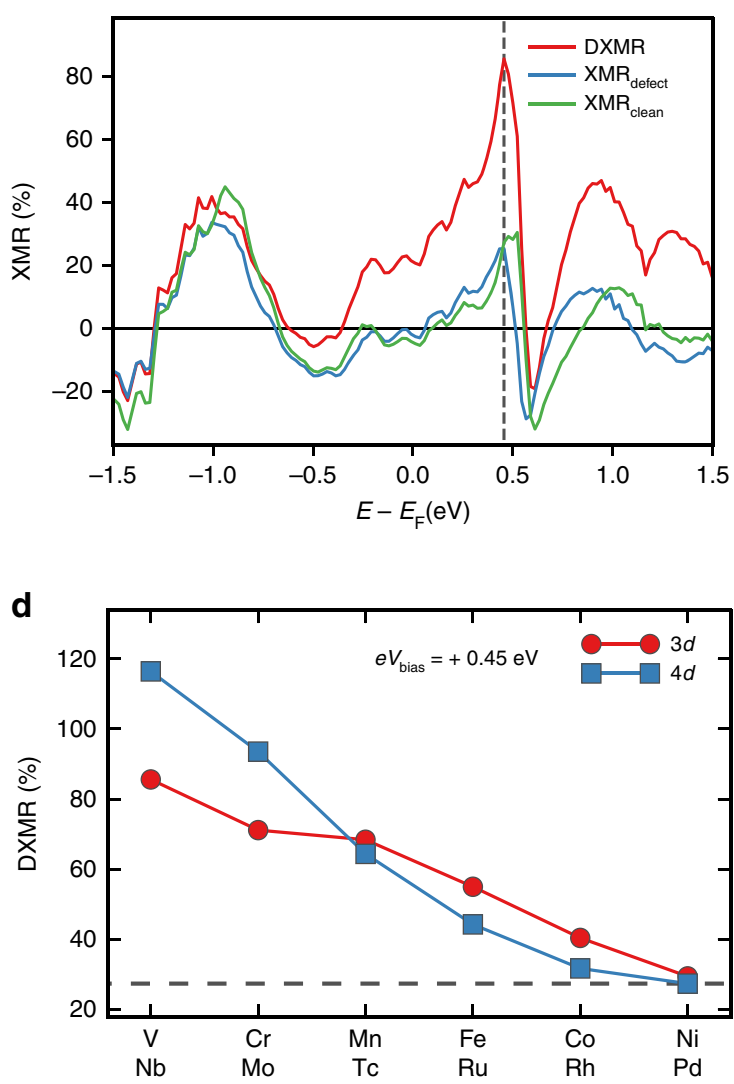

b

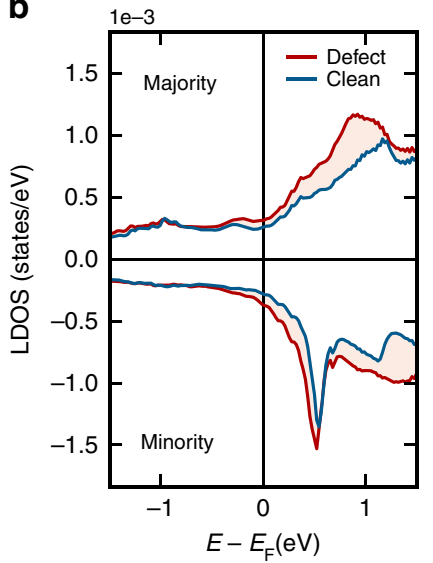

e

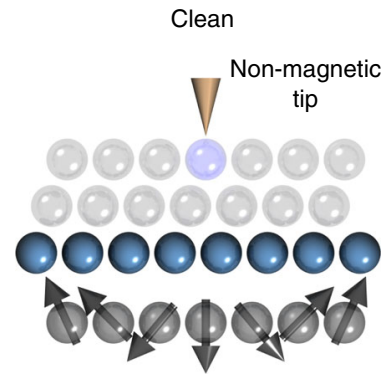

c

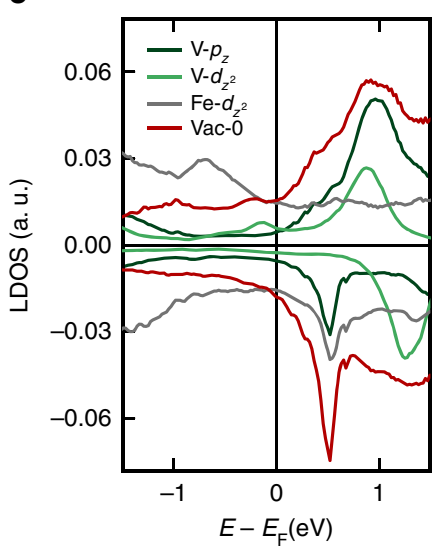

f

Defect

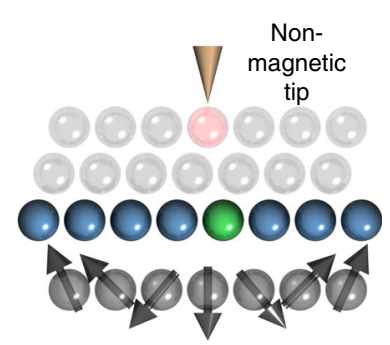

Fig. 2 Efficiency of the XMR signal and the electronic structure. a Comparison of various energy-resolved XMR signals measured on top of the skyrmion's core with $\mathrm{V}$ impurity located close to skyrmion's core. The dashed line indicates the energy at which the DXMR reaches the maximum efficiency. b Electronic structure in the vacuum on top of the skyrmion's core resolved into majority and minority spin-channels for the clean system (blue line) and with a $\mathrm{V}$-impurity (red line). c Superposition of the electronic states in vacuum of $\mathrm{V}$ and Fe at the skyrmion's core. Note that arbitrary units are used to include all of the curves in the same plot. $\mathbf{d}$ Impact of chemical nature of the defect on the DXMR signal at an injection energy $\mathrm{eV}$ bias $=0.45 \mathrm{eV}$ for $3 d$ (red circles) and $4 d$ (blue squares) impurities located close to the skyrmion center. The gray dashed line indicates the signal of the $X M R_{c l e a n}$. $\mathbf{e}, \mathbf{f} \| l$ ustrative legend for the STM-tip probing the clean (defective) substrate at the location defined by the light blue (red) sphere, whose color corresponds to the LDOS plotted in (b). Vacuum is simulated by empty cells, schematically represented as spheres.

a substrate hosting impurities. The overall STM/STS signal is increased, which greatly facilitates the detection of buried skyrmions, as it is for PdFe/ $\operatorname{Ir}(111)$ surface. Thus, defects can act as mediating probes for the electrical detection. The observed vacuum states originate from the decay of the electronic states of the V-defect and Fe layer plotted in Fig. 2c, as can be noticed from the one-to-one correspondence between the atomic virtual bound states and the features probed in vacuum. The orbitalresolved LDOS, e.g., the $p_{\mathrm{z}}$ and $d_{\mathrm{z}^{2}}$ states, leading to the largest tunneling matrix elements are shown in particular for the $\mathrm{V}$ defect and for the Fe atom at the skyrmion's core. The latter defines the spin-frame of reference in which the LDOS is plotted and the convention to denote the majority- and minority-spin states.

Because of the large exchange splitting of $\mathrm{V}$, the hybridization is weak between the occupied majority-spin states of Fe and the unoccupied ones of $\mathrm{V}$, giving rise to the $d_{\mathrm{z}^{2}}$ and $p_{\mathrm{z}}$ virtual bound states located at $\approx+0.9 \mathrm{eV}$ and $\approx+1.0 \mathrm{eV}$, respectively (see upper channel of Fig. 2c). Recalling that $\mathrm{V}$ replaces a Pd atom from the defect-free substrate, we expect thus a larger intensity of the features observed in vacuum because of the additional impurity-states. Similar conclusions can be drawn for the opposite spin channel. Interestingly, an unoccupied $p_{\mathrm{z}}$ virtual bound state at $\approx+0.53 \mathrm{eV}$ is induced by the Fe minority-spin state in the LDOS of V (see lower channel of Fig. 2c), with a larger intensity than the one obtained in the clean Pd-overlayer.

Systematic trends. The enhancement of bare transport signal detected within the DXMR signal with respect to the conventional $\mathrm{XMR}$ is not limited to the $\mathrm{V}$ impurity but occurs for all investigated implanted defects of the $3 d$ and $4 d$ series. The factor of enhancement depends, however, on the chemical nature of the impurities as well as on the injection energy. In Fig. $2 \mathrm{~d}$, we systematically collect the efficiency of the DXMR as function of the impurities atomic number for an injection energy of $e V_{\text {bias }}=+0.45 \mathrm{eV}$. Overall, the DXMR ratio decreases when increasing the atomic number of the defects. For the $3 d$ elements, $\mathrm{V}$ leads to the highest efficiency, which is larger than the $30 \%$ efficiency induced by $\mathrm{Ni}$. The latter defect does not alter the defect-free XMR efficiency. Interestingly, defects from the beginning of the $4 d$ series lead to an impressive enhancement of the signal, reaching almost $116 \%$ for $\mathrm{Nb}$ followed by $94 \%$ for Mo which translate to a increase of about $350 \%$ and $260 \%$, respectively, with respect to the defect-free XMR efficiency. This implies that the best impurities for the enhancement of the bare tunneling transport signal are early transition elements with a preference for the $4 d$ series. 
a

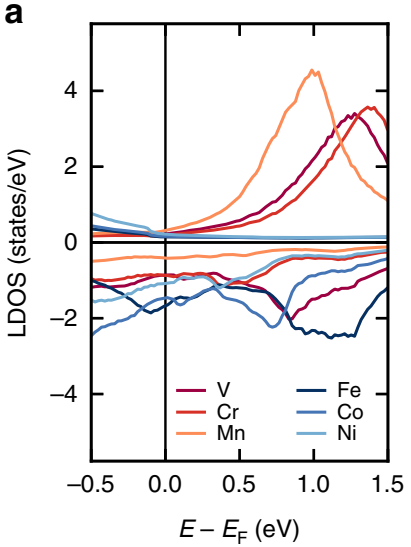

e

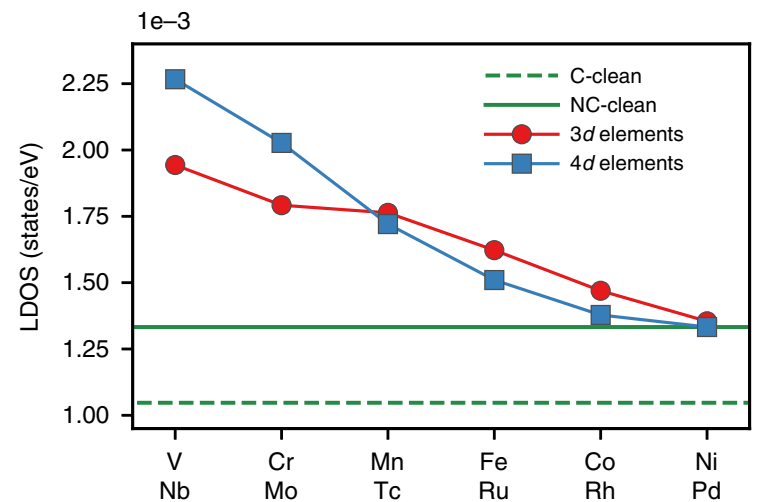

b
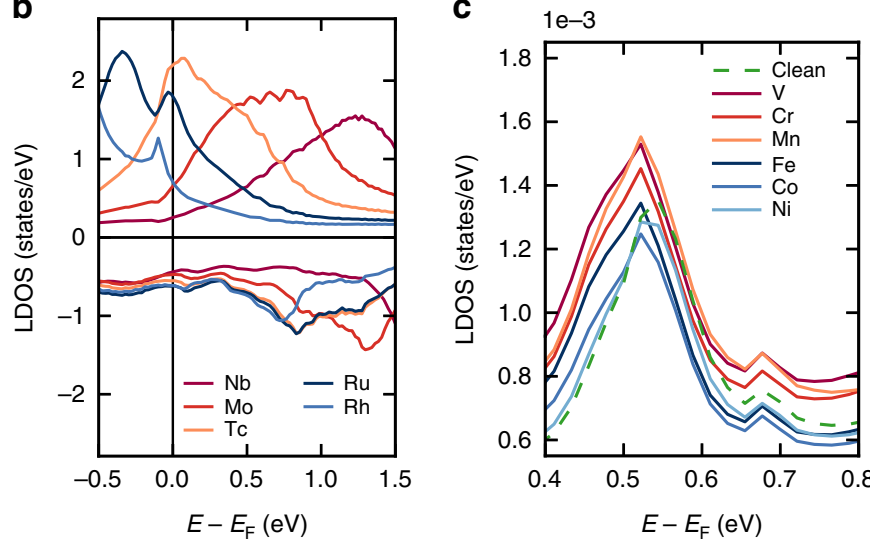

d $1 \mathrm{e}-3$

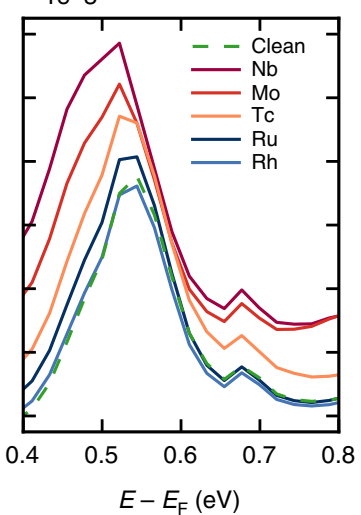

$\mathbf{f}$

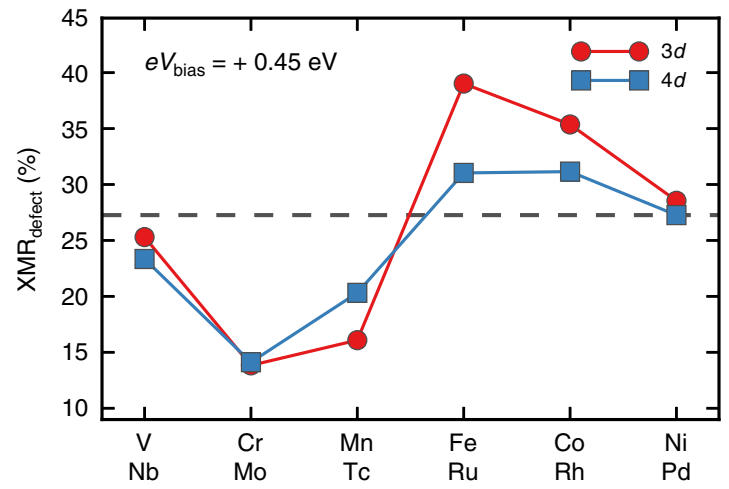

Fig. 3 Impact of defects on the electronic structure and on the $\mathbf{X} \mathbf{M} \mathbf{R}_{\text {defect }}$ signal. Local density of states (LDOS) of the defect within the collinear configuration for $\mathbf{a}$ the $3 d$-inatom series $\mathbf{b}$ the $4 d$-inatom series. The minority-spin channels of the LDOS of the vacuum on top of the core of the skyrmion with a c $3 d$ and $\mathbf{d} 4 d$ impurity close to the skyrmion core. e Chemical trend of the total LDOS of the vacuum on top of the core of the skyrmion at +0.45 eV for the $3 d$-elements (red line) $4 d$-elements (blue line), where the total LDOS is the sum of the LDOS of the minority- and majority-spin states. The green full line (dashed line) indicates the LDOS of the vacuum within the non-collinear (collinear) magnetic region for the defect free-system substrate at +0.45 $\mathrm{eV}$. $\mathbf{f}$ Impact of band-filling on the defect spin-mixing magnetoresistance $\left(\mathrm{XMR}_{\mathrm{defect}}\right)$ at an injection energy eV $V_{\text {bias }}=0.45$ eV. The gray dashed line indicates the signal of the $X M R_{\text {clean }}$.

The behavior of the DXMR signal as function of the chemical nature of the defect can be directly related to the filling of the impurities electronic states. By moving from left to right across the $3 d(4 d)$ atomic row of the periodic table the unoccupied states of the impurities shift towards the Fermi energy becoming partially or almost fully occupied at the end of the series as can be seen in Fig. 3a, b for the collinear magnetic states. Interestingly, this sequence is accompanied with a transition from an antiferromagnetic coupling to the substrate for $\mathrm{V}(\mathrm{Nb}), \mathrm{Cr}(\mathrm{Mo})$, and $\mathrm{Mn}(\mathrm{Tc})$ to a ferromagnetic coupling for $\mathrm{Fe}(\mathrm{Ru})$, Co $(\mathrm{Rh})$, and $\mathrm{Ni}(\mathrm{Pd})$. The decrease of the impurities LDOS upon the filling of the electronic states, as illustrated by Fig. 3e, explains the DXMR trend plotted in Fig. $2 \mathrm{~d}$ as well as the very large ratio induced by the $4 d$ impurities when compared to the $3 d$ ones. The smaller exchange splitting of the $4 d$ elements increases the possibility of having large LDOS around the Fermi energy.

Paying closer attention to the electronic features of the vacuum on top of the core of the skyrmion responsible for the discussed effect for the $3 d$ and $4 d$ series (see Fig. $3 c$, d), one identifies that the peaks obtained for the antiferromagnetic $\mathrm{V}(\mathrm{Nb}), \mathrm{Cr}(\mathrm{Mo})$ and $\mathrm{Mn}(\mathrm{Tc})$ are more intense than those of the ferromagnetic Fe $(\mathrm{Ru}), \mathrm{Co}(\mathrm{Rh})$ and $\mathrm{Ni}(\mathrm{Pd})$. Here, two concomitant mechanisms are at play. First the hybridization strength is known to decrease from left-to-right and to increase from up-to-down across the transition element series of the periodic table ${ }^{37}$. Second, the $p_{z}$ state induced in the electronic structure of the impurities depends on the magnetic coupling because of the switch of the spin-nature of the impurity states hybridizing with the minority-spin states of Fe. Thus, more impurity states are available when the spinalignment is rather ferromagnetic.

Contrasting the DXMR behavior, the $\mathrm{XMR}_{\text {defect }}$ for an injection energy $e V_{\text {bias }}=+0.45 \mathrm{eV}$ when plotted as function of the atomic number (see Fig. 3f) exhibits a S-like shape with a maximum and a minimum close to the middle of the series with the efficiency increasing up to about $56 \%$ with respect to the defect-free signal. At this particular injection energy, the $\mathrm{XMR}_{\text {defect }}$ signal is better magnified by impurities with more than half-filled $d$-states instead of the early transition elements, which are better suited to enhance the bare transport signal as previously discussed.

Figure $4 \mathrm{a}, \mathrm{b}$ shows a comparison of the $\mathrm{XMR}_{\mathrm{defect}}$ and DXMR efficiencies at the injection energy $e V_{\text {bias }}$ of $-1.27 \mathrm{eV}$ for the $3 d$ and $4 d$ defects. One notices that by changing the injection energy, the S-shape observed for $\mathrm{XMR}_{\text {defect }}$ at $0.45 \mathrm{eV}$ can be reversed and the location of the extrema can be strongly shifted. The usual $\mathrm{XMR}_{\text {clean }}$ efficiency is found rather low with a value of $4.6 \%$ but thanks to the defects, the XMR efficiencies increase. Among the $3 d$ impurities, $\mathrm{Fe}$ and $\mathrm{Co}$ induce $\mathrm{XMR}_{\text {defect }}$ efficiencies of respectively $17.4 \%$ and $11.5 \%$, i.e., an increase of respectively $277 \%$ and $151 \%$ with respect to $\mathrm{XMR}_{\text {clean }}$, which are a factor two larger than the DXMR ones of $8.7 \%$ and $5.1 \%$, respectively.

In general, we note that the $\mathrm{XMR}_{\text {defect }}$ signal for the $3 d$ elements keeps the S-shape for most of the bias voltages, while the pattern obtained for the $4 d$ elements can change. An overview on 
a

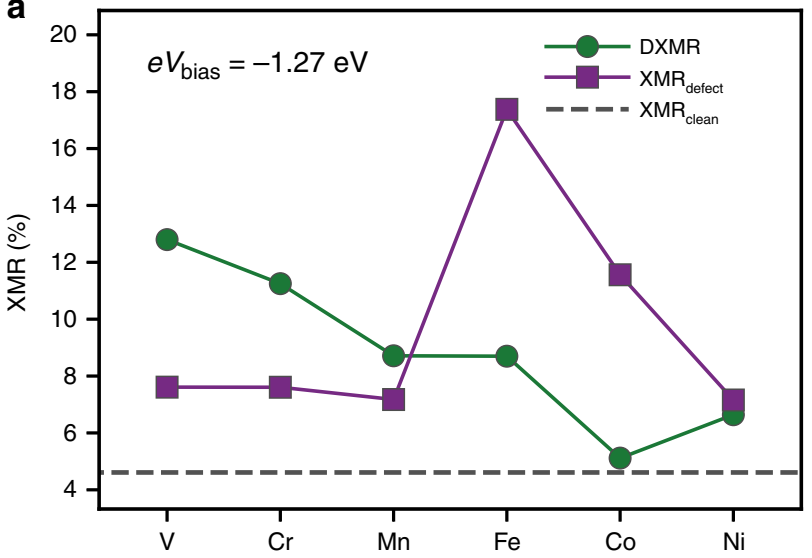

C

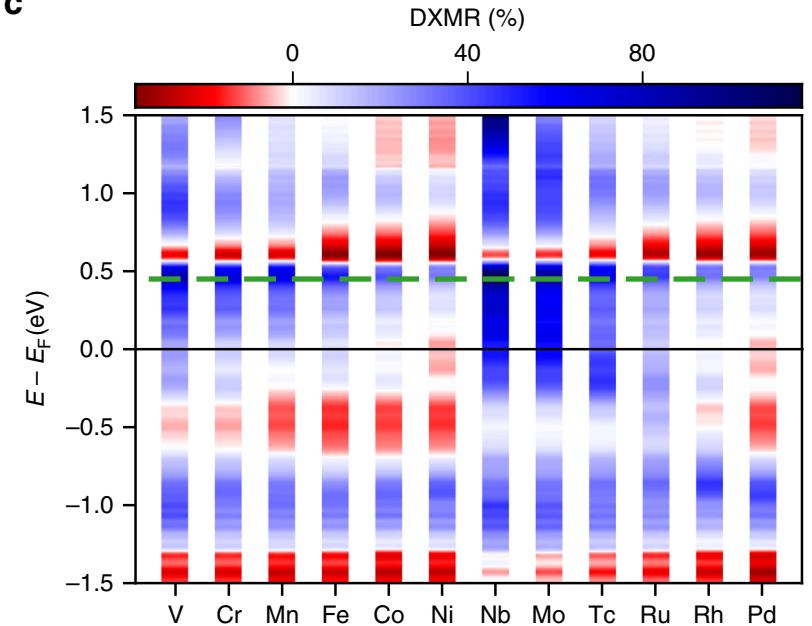

b

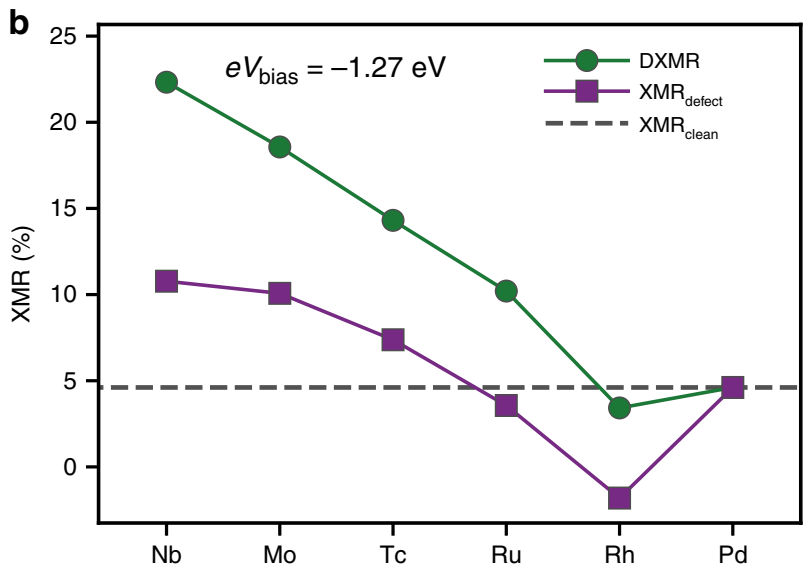

d

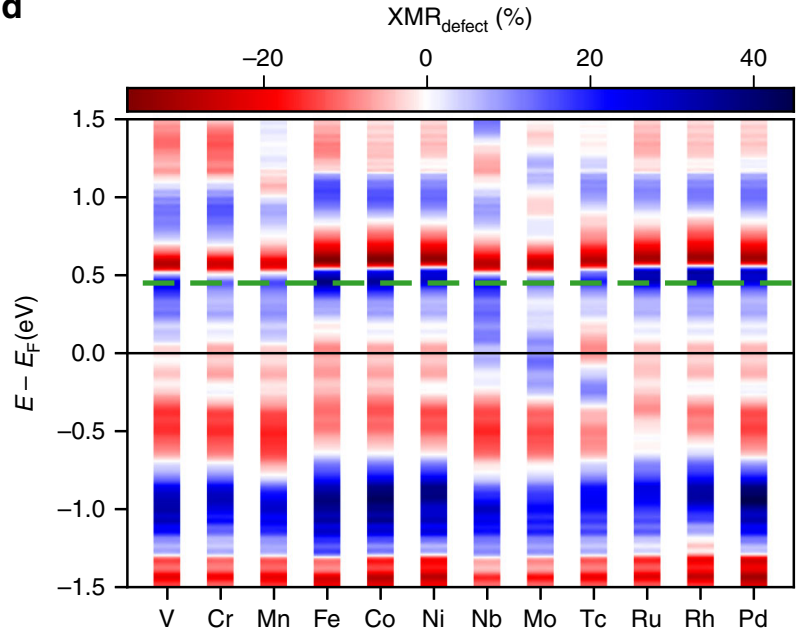

Fig. 4 Impact of the bias voltage on the defect-enabled XMR modes. Dependence of the DXMR signal (green circle) and XMR defect (purple squares), on the atomic number of the $\mathbf{a} 3 d$ and $\mathbf{b} 4 d$ impurities at an injection energy $e V_{\text {bias }}=-1.27 \mathrm{eV}$. The gray dashed line indicates the signal of the $X M R_{c l e a n}$. Bias voltage dependence of the $\mathbf{c} D X M R$ and $\mathbf{d} X M R_{\text {defect }}$ signal for $3 d$ and $4 d$ defects. The color bars indicate the magnitude of the signal and the dashed green line shows the injection energy $\mathrm{eV}$ bias $=0.45 \mathrm{eV}$ studied in Figs. 2 and 3.

the simultaneous dependence of the defects-enabled XMR efficiencies on the atomic number of the impurities and the magnitude of the bias voltage is presented in Fig. 4c, d. The signal corresponding to $\mathrm{XMR}_{\text {clean }}$ is the one of $\mathrm{Pd}$ and the dashed line indicates the particular case of $e V_{\text {bias }}=0.45 \mathrm{eV}$. One notices that the pattern of intensities can be manipulated to a great extent by changing the nature of the defects. There is an effective dispersion of the blue and red "band" having distinct bandwidths depending on the energy or impurities atomic number. Also, one sees how the white "bands", i.e., those corresponding to a weak XMR signal, are modified. Early transition elements seem to generate most of the widening of the blue bands, i.e. large DXMR efficiencies with a positive sign. Interestingly white bands emerge within the large blue bands characterizing the $4 d$ elements. A large difference between the patterns obtained for positive and negative bias voltages is observed for $\mathrm{XMR}_{\text {defect, }}$, with more bluered sequences observed at positive energies. This is probably due to the minority-spin states of the substrate that are more sensitive to the hybridization with the impurity states.

Real-space XMRs contrast and inhomogeneous noncollinearity. The XMR efficiency can be of use to detect slight changes in the magnetic texture. The $\mathrm{XMR}_{\text {clean }}$ and $\mathrm{XMR}_{\text {defect }}$ depend on the opening angle between neighboring magnetic moments. In the defect-free region, the measurable electrical contrast is expected to exhibit a highly symmetric shape, translating the symmetry of the magnetic texture, with the highest intensity at the center of the skyrmion (see Fig. 5a). At the vicinity of a defect, the skyrmion experiences an asymmetric environment impacting its spin-texture and consequently the all-electrical XMR contrast. This is better grasped by visualizing the difference $\Delta \mathrm{XMR}_{\text {defect }}=\mathrm{XMR}_{\text {defect }}-\mathrm{XMR}_{\text {clean }}$ as done in Fig. 5b, $\mathrm{c}$ for $\mathrm{Cr}$ and $\mathrm{Ni}$ impurities. For $\mathrm{Cr}$, the $\mathrm{XMR}_{\text {defect }}$ signal strongly decreases at the close vicinity of the impurity since the strong exchange coupling of the impurity and the substrate atoms reduces the surrounding non-collinearity (see Supplementary Note 3 and Fig. 5e), which lowers the XMR ratio. For $\mathrm{Ni}$, however, the change in the XMR signal (Fig. $5 \mathrm{c}$ ) is off-set from the defect with the pattern being less intense than for $\mathrm{Cr}$, which corresponds to the modifications induced in the magnetic texture shown in Fig. 5f. We note that in Fig. 5e, f the noncollinearity is probed in terms of the topological charge density (more details are given in Supplementary Note 4). For the investigated bias voltage of $0.45 \mathrm{eV}$, the DXMR signal tend to be not that sensitive to the impurity-induced non-collinear modifications and reaches a maximum value on top of all defects. As an example, the Cr-related DXMR signal at the same bias energy is illustrated in Fig. 5d when the impurity is close to the core of the skyrmion. 
a

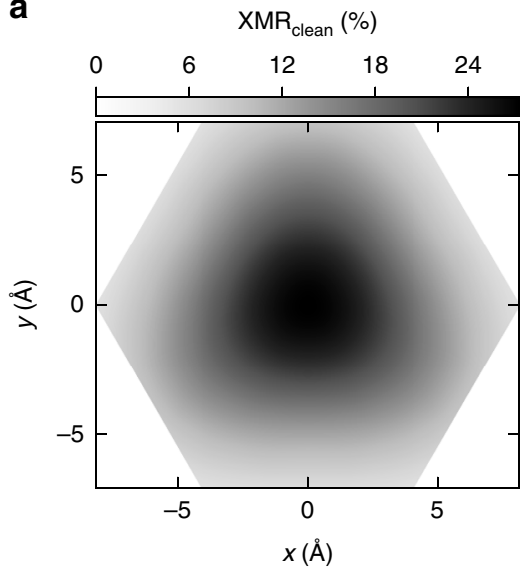

d

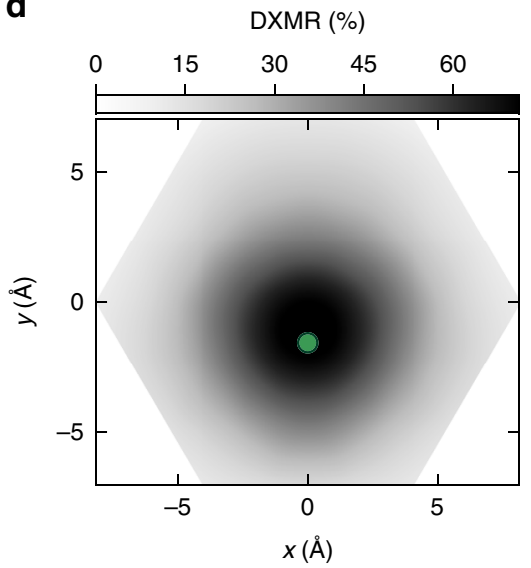

b

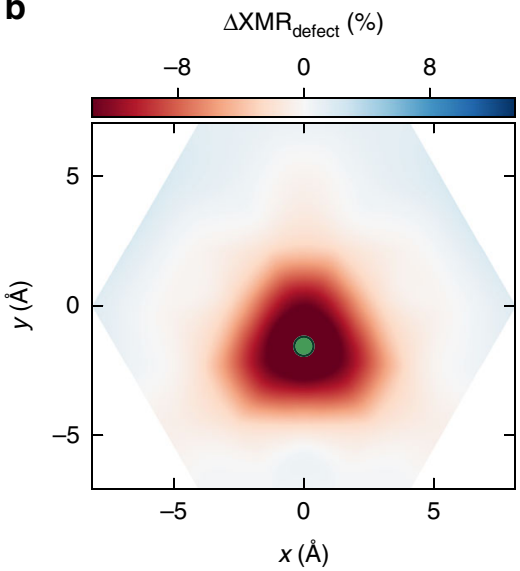

e

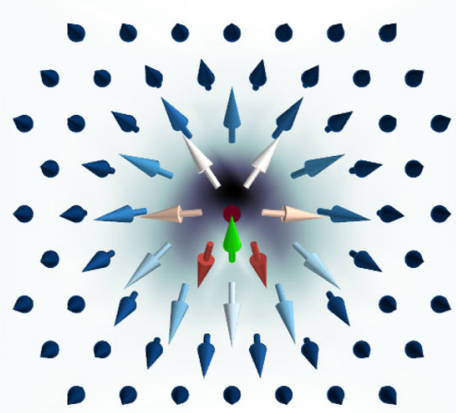

$M_{z}\left(\mu_{\mathrm{B}}\right)$

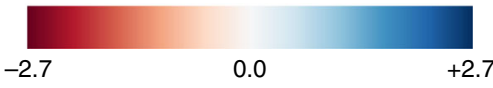

C

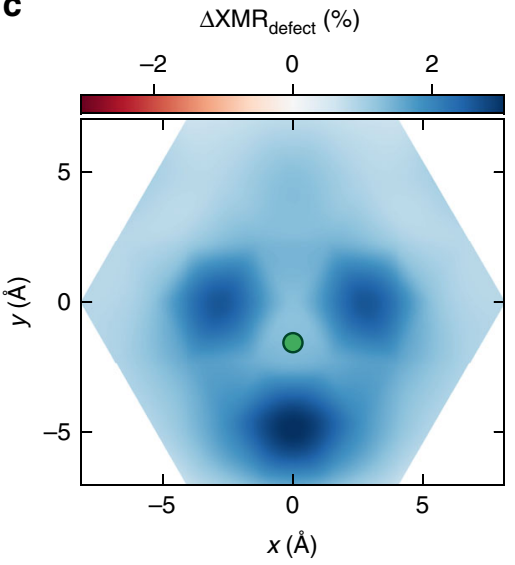

$\mathbf{f}$

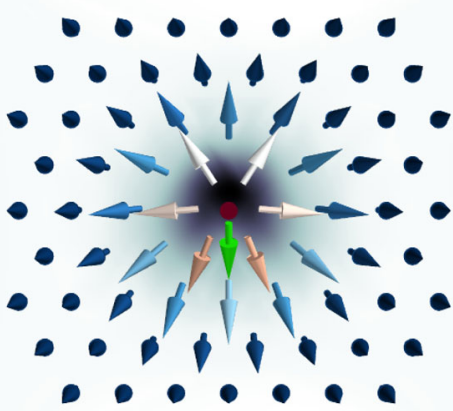

9

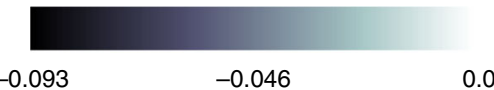

Fig. 5 Impact of single-atomic defects on XMR-signals of skyrmions. a Defect-free XMR signal compared to $\mathbf{d}$ DXMR signal induced by a Cr impurity. Difference between $X M R_{\text {defect }}$ and $X M R_{\text {clean }}$ plotted for $\mathbf{b} \mathrm{Cr}$ and $\mathbf{c}$ Ni impurities close to the core of skyrmion. The green circle indicates the position of the impurity. The corresponding skyrmion magnetic textures and the topological charge monitoring the non-collinearity are shown in (e) for $\mathrm{Cr}$ and (f) for $\mathrm{Ni}$, where the green arrow represents the impurity magnetic moment while the rest of arrows are those of the Fe atoms. The red-blue color bar represents the magnitude of the out-of-plane magnetization $\left(M_{z}\right)$ of each Fe atom while the grayscale color bar is used for the topological charge computed for triangles defined by sets of three nearest neighboring atoms. An interpolation is then performed between the discrete values of the charge.

\section{Discussion}

In this work, we explore the possibility of using defects to detect non-collinear magnetic textures using all-electrical means. By strongly modifying the tunneling matrix elements, defects can increase the intensity of the background transport signal. This would prove useful in case of buried non-collinear spin-textures, which are difficult to access with surface probe techniques. To track the impact of impurities, we defined the $\mathrm{XMR}_{\text {defect }}$ and DXMR, which monitor the enhancement of the XMR efficiency induced by the presence of the defects. DXMR can even benefit from the amplification provided by the modification of the bare transport signal. Since both proposed defect-enabled XMR modes could be simultaneously accessed, it is expected that one can select the one with the best efficiency while performing the measurements.

From the obtained general trends upon filling of the impurities electronic states, our investigations at the bias voltage of $e V_{\text {bias }}=$ $+0.45 \mathrm{eV}$ point to the use of early transition elements embedded in $\mathrm{Pd} / \mathrm{Fe} / \mathrm{Ir}(111)$ to enhance the bare tunneling transport patterns, while elements with more than half-filled $d$-shells seem to be better suited for a better detection via $\mathrm{XMR}_{\text {defect }}$. Besides the atomic number of the impurities, the applied bias voltage is an important parameter for the various effects shaping the XMR efficiencies, which can modify the general trends to a great extent.

The Tersoff-Hamann approximation ${ }^{52}$ considered for the evaluation of the differential conductance assumes a tip with an $s$-orbital. Changing the nature of the tip-orbitals modifies the tunneling matrix elements dictating which substrate's states contribute to transport ${ }^{53,54}$. The bare transport spectra, and therefore the various $\mathrm{XMR}$, are expected to be altered, which opens a large amount of possibilities to tune the efficiencies of the signals. Obviously, initially "silent" substrate's states can become active and enhance the all-electrical detection abilities by engineering the nature of the probing electrode. This, in principle, can be done by changing either the tip or by deliberately transferring an atom or a molecule to the tip ape $\mathrm{x}^{55-58}$.

The magnitude of the defect-enabled XMR efficiencies hinges on the location of the impurities on the substrate. For instance, the defects can be located atop, instead of being embedded in the $\mathrm{Pd}$ layer as assumed in the current investigation. Our proof-ofconcept study has, however, predictive value since the related formation energies (see Supplementary Note 5) indicate that all investigated $4 d$ impurities and a couple of $3 d$ atoms $(\mathrm{V}, \mathrm{Cr})$ have a clear preference to be embedded in the Pd layer, while Mn and $\mathrm{Ni}$ prefer to sit atop the substrate.

By providing a path to enhance in various ways the allelectrical detection of non-collinear magnetic textures, harnessing defects and their resulting electronic structure might prove useful for applications. We envision single or a complex defects arranged in a controlled manner, as done by STM or ion-implantation, to enhance in specific regions the electrical detection. Thus, the 
DXMR and $\mathrm{XMR}_{\text {defect }}$ techniques can be incorporated in future reading technologies, which enforces the view that controlled engineering of defective-materials is a promising route for device architectures.

\section{Methods}

Computational details. The electronic structure was determined employing first principles calculations based on density functional theory (DFT) in the local spin density approximation. The calculations were performed with the full-potential scalar-relativistic Korringa-Kohn-Rostoker (KKR) Green function method with spin-orbit coupling included self-consistently ${ }^{59,60}$. The method allows to embed single magnetic skyrmions and defects without the need for periodic supercells. This is performed in a two-steps approach. First, self-consistent calculations of the defect-free and skyrmion-free magnetic slab with periodic boundary conditions are done. Afterwards, the Green function, $G_{0}$, of the ferromagnetic substrate is harvested in order to solve the Dyson equation, schematically written as $G=G_{0}-$ $G_{0} \Delta V G$, in order to obtain the new Green function $G . \Delta V$ describes the change in the potential after embedding the perturbation, which as mentioned earlier consists of a non-collinear spin-texture such as a magnetic skyrmion, an impurity or both. The magnetic textures are obtained in a self-consistent fashion till convergence is achieved.

In practice, the $\mathrm{Pd} / \mathrm{Fe} / \mathrm{Ir}(111)$ slab consists of a fcc-stacked PdFe bilayer deposited on 34 layers of Ir with atomic positions obtained from ab initio ${ }^{44}$. The embedded cluster consists of 124 atoms in total among which $37 \mathrm{Fe}$ atoms (see refs. ${ }^{23,37}$ and references therein for details). We assume an angular momentum cutoff at $l_{\max }=3$ for the orbital expansion of the Green function and when extracting the LDOS a k-mesh of $200 \times 200$ is considered.

\section{Data availability}

The data that support the findings of this study are available from the corresponding authors on request.

\section{Code availability}

The KKR Green function code that supports the findings of this study is available from the corresponding author on request.

Received: 27 June 2019; Accepted: 4 March 2020;

Published online: 30 March 2020

\section{References}

1. Wolf, S. et al. Spintronics: a spin-based electronics vision for the future. Science 294, 1488-1495 (2001).

2. Evetts, J. et al. Defect-induced spin disorder and magnetoresistance in singlecrystal and polycrystal rare-earth manganite thin films. Philos. Trans. R. Soc. London A: Math, Phys. Eng. Sci. 356, 1593-1615 (1998).

3. Ke, Y., Xia, K. \& Guo, H. Oxygen-vacancy-induced diffusive scattering in fe/ mgo/fe magnetic tunnel junctions. Phys. Rev. Lett. 105, 236801 (2010).

4. Baibich, M. N. et al. Giant magnetoresistance of (001) Fe/(001) Cr magnetic superlattices. Phys. Rev. Lett. 61, 2472 (1988).

5. Binasch, G., Grünberg, P., Saurenbach, F. \& Zinn, W. Enhanced magnetoresistance in layered magnetic structures with antiferromagnetic interlayer exchange. Phys. Rev. B 39, 4828 (1989).

6. Julliere, M. Tunneling between ferromagnetic films. Phys. Lett. A 54, 225-226 (1975).

7. Moodera, J. S., Kinder, L. R., Wong, T. M. \& Meservey, R. Large magnetoresistance at room temperature in ferromagnetic thin film tunnel junctions. Phys. Rev. Lett. 74, 3273 (1995).

8. Garcia, N., Munoz, M. \& Zhao, Y.-W. Magnetoresistance in excess of $200 \%$ in ballistic Ni nanocontacts at room temperature and 100 Oe. Phys. Rev. Lett. 82, 2923 (1999).

9. Tatara, G., Zhao, Y.-W., Munoz, M. \& Garcia, N. Domain wall scattering explains 300\% ballistic magnetoconductance of nanocontacts. Phys. Rev. Lett. 83, 2030 (1999).

10. Palotás, K., Lazarovits, B., Szunyogh, L. \& Weinberger, P. Ab initio study of the electric transport in gold nanocontacts containing single impurities. Phys. Rev. B 70, 134421 (2004)

11. Néel, N. et al. Tunneling anisotropic magnetoresistance at the single-atom limit. Phys. Rev. Lett. 110, 037202 (2013)

12. Stipe, B. C., Rezaei, M. A. \& Ho, W. Single-molecule vibrational spectroscopy and microscopy. Science 280, 1732-1735 (1998).

13. Heinrich, A. J., Gupta, J. A., Lutz, C. P. \& Eigler, D. M. Single-atom spin-flip spectroscopy. Science 306, 466-469 (2004).
14. Schweflinghaus, B., dosSantosDias, M., Costa, A. T. \& Lounis, S Renormalization of electron self-energies via their interaction with spin excitations: A first-principles investigation. Phys. Rev. B 89, 235439 (2014).

15. Nagaosa, N., Sinova, J., Onoda, S., MacDonald, A. H. \& Ong, N. P. Anomalous hall effect. Rev. Mod. Phys. 82, 1539-1592 (2010)

16. Gradhand, M., Fedorov, D. V., Zahn, P. \& Mertig, I. Extrinsic spin hall effect from first principles. Phys. Rev. Lett. 104, 186403 (2010).

17. Lowitzer, S. et al. Extrinsic and intrinsic contributions to the spin hall effect of alloys. Phys. Rev. Lett. 106, 056601 (2011).

18. Fert, A. \& Levy, P. M. Spin hall effect induced by resonant scattering on impurities in metals. Phys. Rev. Lett. 106, 157208 (2011).

19. Zimmermann, B., Long, N. H., Mavropoulos, P., Blügel, S. \& Mokrousov, Y. Influence of complex disorder on skew-scattering Hall effects in $L 1_{0}$-ordered FePt alloy. Phys. Rev. B 94, 060406 (2016).

20. Bouaziz, J., Lounis, S., Blügel, S. \& Ishida, H. Microscopic theory of the residual surface resistivity of rashba electrons. Phys. Rev. B 94, 045433 (2016).

21. Kondo, J. Resistance minimum in dilute magnetic alloys. Prog. Theor. Exp. Phys. 32, 37-49 (1964).

22. Daybell, M. \& Steyert, W. Localized magnetic impurity states in metals: Some experimental relationships. Rev. Mod. Phys. 40, 380 (1968).

23. Crum, D. M. et al. Perpendicular reading of single confined magnetic skyrmions. Nat. Commun. 6, 8541 (2015).

24. Hanneken, C. et al. Electrical detection of magnetic skyrmions by tunnelling non-collinear magnetoresistance. Nat. Nanotechnol. 10, 1039 (2015).

25. Bogdanov, A. N. \& Yablonskii, D. Thermodynamically stable "vortices" in magnetically ordered crystals. the mixed state of magnets. J. Exp. Theor. Phys. 95, 178 (1989).

26. Rössler, U. K., Bogdanov, A. N. \& Pfleiderer, C. Spontaneous skyrmion ground states in magnetic metals. Nature 442, 797-801 (2006).

27. Fert, A., Reyren, N. \& Cros, V. Magnetic skyrmions: advances in physics and potential applications. Nat. Rev. Mater. 2, 17031 (2017).

28. Fert, A., Cros, V. \& Sampaio, J. Skyrmions on the track. Nat. Nanotech. 8, 152-156 (2013)

29. Sampaio, J., Cros, V., Rohart, S., Thiaville, A. \& Fert, A. Nucleation, stability and current-induced motion of isolated magnetic skyrmions in nanostructures. Nat. Nanotech. 8, 839-844 (2013).

30. Iwasaki, J., Mochizuki, M. \& Nagaosa, N. Universal current-velocity relation of skyrmion motion in chiral magnets. Nat. Commun. 4, 1463 (2013).

31. Iwasaki, J., Mochizuki, M. \& Nagaosa, N. Current-induced skyrmion dynamics in constricted geometries. Nat. Nanotech. 8, 742-747 (2013).

32. Jiang, W. et al. Blowing magnetic skyrmion bubbles. Science 349, 283-286 (2015).

33. Woo, S. et al. Observation of room-temperature magnetic skyrmions and their current-driven dynamics in ultrathin metallic ferromagnets. Nat. Mater. 15, 501-506 (2016).

34. Hanneken, C., Kubetzka, A., vonBergmann, K. \& Wiesendanger, R. Pinning and movement of individual nanoscale magnetic skyrmions via defects. New J. Phys. 18, 055009 (2016).

35. Jiang, W. et al. Direct observation of the skyrmion Hall effect. Nat. Phys. 13, 162-169 (2017).

36. Litzius, K. et al. Skyrmion hall effect revealed by direct time-resolved $\mathrm{x}$-ray microscopy. Nat. Phys. 13, 170-175 (2017).

37. Lima Fernandes, I., Bouaziz, J., Blügel, S. \& Lounis, S. Universality of defectskyrmion interaction profiles. Nat. Commun. 9, 4395 (2018).

38. Choi, H. C., Lin, S.-Z. \& Zhu, J.-X. Density functional theory study of skyrmion pinning by atomic defects in mnsi. Phys. Rev. B 93, 115112 (2016).

39. Maccariello, D. et al. Electrical detection of single magnetic skyrmions in metallic multilayers at room temperature. Nat. Nanotechnol. 13, 233 (2018).

40. Eigler, D. M. \& Schweizer, E. K. Positioning single atoms with a scanning tunnelling microscope. Nature 344, 524-526 (1990).

41. Persaud, A. et al. Integration of scanning probes and ion beams. Nano Lett. 5 , 1087-1091 (2005)

42. Romming, N. et al. Writing and deleting single magnetic skyrmions. Science 341, 636-639 (2013).

43. Romming, N., Kubetzka, A., Hanneken, C., vonBergmann, K. \& Wiesendanger, R. Field-dependent size and shape of single magnetic skyrmions. Phys. Rev. Lett. 114, 177203 (2015).

44. Dupé, B., Hoffmann, M., Paillard, C. \& Heinze, S. Tailoring magnetic skyrmions in ultra-thin transition metal films. Nat. Commun. 5, 4030 (2014).

45. Simon, E., Palotás, K., Rózsa, L., Udvardi, L. \& Szunyogh, L. Formation of magnetic skyrmions with tunable properties in PdFe bilayer deposited on $\mathrm{Ir}$ (111). Phys. Rev. B 90, 094410 (2014).

46. Leonov, A. et al. The properties of isolated chiral skyrmions in thin magnetic films. New J. Phys. 18, 065003 (2016).

47. dosSantosDias, M., Bouaziz, J., Bouhassoune, M., Blügel, S. \& Lounis, S. Chirality-driven orbital magnetic moments as a new probe for topological magnetic structures. Nat. Commun. 7, 13613 (2016). 
48. Dzyaloshinsky, I. A thermodynamic theory of "weak" ferromagnetism of antiferromagnetics. J. Phys. Chem. Sol. 4, 241-255 (1958).

49. Moriya, T. Anisotropic superexchange interaction and weak ferromagnetism. Phys. Rev. 120, 91-98 (1960).

50. Kubetzka, A., Hanneken, C., Wiesendanger, R. \& vonBergmann, K. Impact of the skyrmion spin texture on magnetoresistance. Phys. Rev. B 95, 104433 (2017).

51. Bouhassoune, M., Fernandes, I. L., Blügel, S. \& Lounis, S. Unoccupied surface and interface states in Pd thin films deposited on Fe/Ir (111) surface. New J. Phys. 21, 063015 (2019).

52. Tersoff, J. \& Hamann, D. Theory and application for the scanning tunneling microscope. Phys. Rev. Lett. 50, 1998 (1983).

53. Chen, C. J. Tunneling matrix elements in three-dimensional space: The derivative rule and the sum rule. Phys. Rev. B 42, 8841-8857 (1990).

54. Chen, C. J. Origin of atomic resolution on metal surfaces in scanning tunneling microscopy. Phys. Rev. Lett. 65, 448-451 (1990).

55. Bartels, L., Meyer, G. \& Rieder, K.-H. Controlled vertical manipulation of single co molecules with the scanning tunneling microscope: a route to chemical contrast. Appl. Phys. Lett. 71, 213-215 (1997).

56. Repp, J., Meyer, G., Stojković, S. M., Gourdon, A. \& Joachim, C. Molecules on insulating films: scanning-tunneling microscopy imaging of individual molecular orbitals. Phys. Rev. Lett. 94, 026803 (2005).

57. Palotás, K., Hofer, W. A. \& Szunyogh, L. Theoretical study of the role of the tip in enhancing the sensitivity of differential conductance tunneling spectroscopy on magnetic surfaces. Phys. Rev. B 83, 214410 (2011).

58. Esat, T., Friedrich, N., Tautz, F. S. \& Temirov, R. A standing molecule as a single-electron field emitter. Nature 558, 573-576 (2018).

59. Papanikolaou, N., Zeller, R. \& Dederichs, P. H. Conceptual improvements of the KKR method. J. Phys. Condens. Matter 14, 2799 (2002).

60. Bauer, D. S. G. Development of a relativistic full-potential first-principles multiple scattering green function method applied to complex magnetic textures of nano structures at surfaces. PhD Dissertation at the RWTH-Aachen (2013).

\section{Acknowledgements}

This work is supported by the European Research Council (ERC) under the European Union's Horizon 2020 research and innovation programme (ERC-consolidator grant 681405 DYNASORE). We acknowledge the computing time granted by the JARA-HPC Vergabegremium and VSR commission on the supercomputers JURECA at Forschungszentrum Jülich and at the supercomputing centre of RWTH Aachen University.

\section{Author contributions}

S.L. initiated, designed and supervised the project. I.L.F. performed the first-principles calculations and post-processed the data. I.L.F., M.B., and S.L. discussed the results and helped writing the manuscript.

\section{Competing interests}

The authors declare no competing interests.

\section{Additional information}

Supplementary information is available for this paper at https://doi.org/10.1038/s41467020-15379-6.

Correspondence and requests for materials should be addressed to I.L.F. or S.L.

Peer review information Nature Communications thanks Krisztian Palotas, Jian-Xin Zhu and the other, anonymous, reviewer for their contribution to the peer review of this work.

Reprints and permission information is available at http://www.nature.com/reprints

Publisher's note Springer Nature remains neutral with regard to jurisdictional claims in published maps and institutional affiliations.

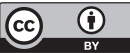

Open Access This article is licensed under a Creative Commons Attribution 4.0 International License, which permits use, sharing, adaptation, distribution and reproduction in any medium or format, as long as you give appropriate credit to the original author(s) and the source, provide a link to the Creative Commons license, and indicate if changes were made. The images or other third party material in this article are included in the article's Creative Commons license, unless indicated otherwise in a credit line to the material. If material is not included in the article's Creative Commons license and your intended use is not permitted by statutory regulation or exceeds the permitted use, you will need to obtain permission directly from the copyright holder. To view a copy of this license, visit http://creativecommons.org/ licenses/by/4.0/.

(C) The Author(s) 2020 